\title{
Avaliação de lesões por serra circular em um centro de referência em microcirurgia e reimplantes*
}

\section{Evaluation of Circular Saw Injuries in a Reference Center in Microsurgery and Reimplantation}

\author{
Ana Lúcia Campos Faccioni ${ }^{1}$ \\ Marcelo Rosa de Rezende ${ }^{4 \odot}$ \\ 1 Programa de Prática Profissionalizante em Microcirurgia, Instituto de \\ Ortopedia e Traumatologia, Hospital das Clínicas, Faculdade de Medicina, \\ Universidade de São Paulo (IOT-HCFMUSP), São Paulo, SP, Brasil \\ 2 Residência Médica em Cirurgia da Mão e Microcirurgia, Instituto de \\ Ortopedia e Traumatologia, Hospital das Clínicas, Faculdade de Medicina, \\ Universidade de São Paulo (IOT-HCFMUSP), São Paulo, SP, Brasil \\ ${ }^{3}$ Departamento de Assistência Social, Instituto de Ortopedia e \\ Traumatologia, Hospital das Clínicas, Faculdade de Medicina, \\ Universidade de São Paulo (IOT-HCFMUSP), São Paulo, SP, Brasil \\ ${ }^{4}$ Grupo de Cirurgia da Mão e Microcirurgia, Instituto de Ortopedia e \\ Traumatologia, Hospital das Clínicas, Faculdade de Medicina, \\ Universidade de São Paulo (IOT-HCFMUSP), São Paulo, SP, Brasil
}

Leonardo Kurebayashi ${ }^{20}$ Kátia Campos dos Anjos ${ }^{3}$

Endereço para correspondência Ana Lúcia Campos Faccioni, MD, Instituto de Ortopedia e Traumatologia, Hospital das Clínicas, Rua Dr. Ovídio Pires de Campos, 333, Cerqueira Cesar, São Paulo, SP, 05403010, Brasil (e-mail: ana.faccioni@gmail.com).

\section{Resumo}

\section{Palavras-chave}

- traumatismos da mão/etiologia

- traumatismos da mão/epidemiologia

- traumatismos da mão/prevenção e controle

- acidentes
Objetivo Avaliar o perfil epidemiológico, o tempo até o atendimento, e o tipo de conduta tomada em pacientes vítimas de acidentes com serra circular e lesões decorrentes, e fazer uma comparação com a literatura.

Métodos Estudo descritivo transversal, com revisão do prontuário de pacientes atendidos de abril a dezembro de 2018, analisando idade, sexo, lado lesado, dedos acometidos, mês e horário do acidente, tipo de lesões, procedimentos realizados na urgência, tempo decorrido entre o trauma e entrada em sala cirúrgica, e reabordagem durante a internação. Resultados Foram atendidos 54 pacientes do sexo masculino com idade entre 15 e 72 anos. O lado esquerdo foi o mais acometido, e o tipo de lesão mais frequente, a amputação, envolvendo principalmente o polegar. No total, 23 pacientes foram submetidos a reimplante, e, entre eles, 3 macrorreimplantes. Quanto ao horário do trauma, 26 ocorreram entre $12 \mathrm{~h}$ e $16 \mathrm{~h}$, e o tempo decorrido entre o acidente e a entrada em sala cirúrgica foi $\geq 6$ horas em $84 \%$ dos pacientes.

\footnotetext{
Trabalho desenvolvido pelo Grupo de Cirurgia da Mão e Microcirurgia, Instituto de Ortopedia e Traumatologia, Hospital das Clínicas, Faculdade de Medicina, Universidade de São Paulo (IOTHCFMUSP), São Paulo, SP, Brasil.
}

recebido

05 de Agosto de 2020

aceito

01 de Dezembro de 2020

Publicado on-line

Agosto 27, 2021
DOI https://doi.org/

10.1055/s-0041-1729940.

ISSN 0102-3616. (c) 2021. Sociedade Brasileira de Ortopedia e Traumatologia. All rights reserved.

This is an open access article published by Thieme under the terms of the Creative Commons Attribution-NonDerivative-NonCommercial-License, permitting copying and reproduction so long as the original work is given appropriate credit. Contents may not be used for commercial purposes, or adapted, remixed, transformed or built upon. (https://creativecommons.org/ licenses/by-nc-nd/4.0/)

Thieme Revinter Publicações Ltda., Rua do Matoso 170, Rio de Janeiro, RJ, CEP 20270-135, Brazil 


\section{Abstract}

\section{Keywords}

- hand injuries/etiology

- hand injuries/epidemiology

- hand injuries/prevention and control

- accidents
Conclusão As lesões por serra circular são predominantemente graves, com potencial de deixar sequelas permanentes, e acometem principalmente o polegar. A caracterização do tipo de lesão e as condições de atendimento inicial obtidas neste trabalho poderão ajudar na política de prevenção e atendimento a pacientes vítimas de ferimentos por serra circular. Nível de Evidência IV; Série de Casos.

Objective To evaluate the epidemiologic profile, the time until care, and the type of conduct taken regarding patients who are victims of accidents with circular saws and their resulting injuries, and to make a comparison with the literature.

Methods A cross-sectional descriptive study reviewing the medical records of patients cared for from April to December 2018, analyzing age, gender, injured side, affected fingers, month and time of the accident, type of injuries, procedures performed in the emergency room, time elapsed between trauma and admission to the operating room, and reapproach during hospitalization.

Results A total of 54 male patients aged between 15 and 72 years were care for. The left side was more affected, and the most frequent type of lesion involved the thumb, which had to be amputated in many cases. In total, 23 patients underwent reimplantation, 3 of which were macroreimplantations. Regarding the time of trauma, 26 cases occurred between noon and 4 p.m., and the time elapsed between the accident and the admission to the operating room was $\geq 6$ hours in $84 \%$ of the patients.

Conclusion Circular saw lesions are predominantly severe, with a potential for leaving permanent sequelae, and they affect mainly the thumb. The characterization of the type of injury and the initial care conditions obtained in the present study may contribute to the policy of prevention and care of patients who are victims of circular saw injuries. Level of Evidence IV; Case Series.

\section{Introdução}

De acordo com Fikry et al., ${ }^{1}$ os acidentes com serra circular são o padrão clássico de trauma envolvendo a mão em países em desenvolvimento, e são reconhecidos como um flagelo socioeconômico. São lesões graves, relativamente frequentes, e com potencial de deixar sequelas permanentes. ${ }^{1}$

Nos Estados Unidos, em 2001, a US Consumer Product Safety Comission identificou mais de 42 mil lesões por serras circulares de mesa ou bancada. ${ }^{2}$ Anualmente, são estimadas nos Estados Unidos mais de 31 mil lesões por serra circular não relacionadas ao trabalho. ${ }^{3}$ Outros estudos regionais, envolvendo populações específicas, como o de Hassine et al., ${ }^{4}$ na Tunísia, e o de Fikry et al., ${ }^{1}$ no Marrocos, demonstram uma alta frequência desses acidentes e gravidade das lesões, que muitas vezes levam a amputações.

Apesar disso, há pouca informação na literatura mundial, especialmente no Brasil, a respeito de suas particularidades e seu impacto social. É de grande importância obter dados epidemiológicos nacionais sobre o tipo de lesão e os fatores relacionados a esse tipo de trauma.

Portanto, este trabalho visa identificar as principais características dessas lesões, delineando o perfil epidemiológico e a conduta no atendimento desses pacientes.

\section{Materiais e Métodos}

Foi realizado um estudo descritivo transversal, com coleta de dados retrospectiva do prontuário de pacientes atendidos em um hospital quaternário, referência para atendimento de traumas complexos de membro superior.

Foram incluídos 54 pacientes atendidos de abril a dezembro de 2018 com lesões decorrentes de acidentes com serra circular. A pesquisa foi realizada no Prontuário Eletrônico MV (MV Informática Nordeste Ltda., Recife, PE, Brasil) por um único pesquisador, após avaliação e autorização pelo Comitê de Ética do Hospital (CAAE 96464418.5.0000.0068).

As variáveis analisadas foram idade, sexo, escolaridade, ocupação, status ocupacional, lado lesado, dedos acometidos, mês e horário do acidente, tipo de lesões, procedimentos realizados na urgência, tempo decorrido entre o trauma e a entrada em sala cirúrgica, e necessidade de reabordagem durante a internação.

Os dados obtidos foram analisados com o programa Statistical Package for the Social Sciences (SPSS, IBM Corp., Armonk, NY, EUA), por meio do qual se obteve a frequência.

\section{Resultados}

Nos 9 meses compreendidos pelo estudo, foram atendidos 54 pacientes, todos do sexo masculino, com idades entree $15 \mathrm{e}$ 72 anos (média: 46,24 anos; desvio padrão: 13,19 anos). As 


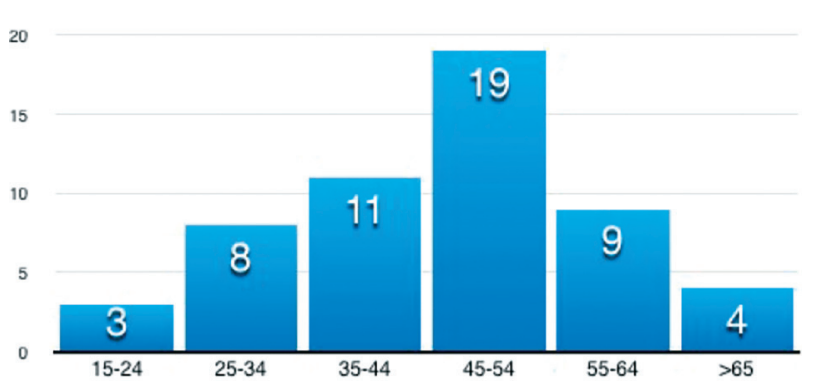

Fig. 1 Número de pacientes por faixa etária.

Tabela 1 Profissões mais envolvidas nos acidentes

\begin{tabular}{|l|l|l|}
\hline Profissão & N & Porcentagem \\
\hline Pedreiro & 16 & $29,6 \%$ \\
\hline Marceneiro & 9 & $26,7 \%$ \\
\hline Ajudante geral & 4 & $7,4 \%$ \\
\hline Aposentado & 3 & $5,6 \%$ \\
\hline Outros & 22 & $30,7 \%$ \\
\hline TOTAL & 54 & $100 \%$ \\
\hline
\end{tabular}

Tabela 2 Tipo de acidente

\begin{tabular}{|l|l|l|}
\hline Tipo de acidente & N & Porcentagem \\
\hline Acidente de trabalho formal & 16 & $29,6 \%$ \\
\hline Acidente de trabalho informal & 21 & $38,9 \%$ \\
\hline Acidente doméstico & 17 & $31,5 \%$ \\
\hline TOTAL & 54 & $100 \%$ \\
\hline
\end{tabular}

Tabela 3 Escolaridade

\begin{tabular}{|l|l|l|}
\hline Escolaridade & $\mathrm{N}$ & Porcentagem \\
\hline $\begin{array}{l}\text { Ensino Fundamental completo } \\
\text { ou incompleto }\end{array}$ & 15 & $27,8 \%$ \\
\hline $\begin{array}{l}\text { Ensino Médio completo } \\
\text { ou incompleto }\end{array}$ & 20 & $37 \%$ \\
\hline Ensino Técnico & 1 & $1,9 \%$ \\
\hline Não informado & 16 & $29,6 \%$ \\
\hline Superior & 2 & $3,7 \%$ \\
\hline TOTAL & 54 & $100 \%$ \\
\hline
\end{tabular}

faixas etárias mais acometidas foram entre 45 e 54 anos (19 pacientes; 35,2\%), de 35 a 44 anos (11 pacientes; 20,4\%), e de 55 a 64 anos (9 pacientes; 16,7\%) (- Figura 1 ).

A profissão mais envolvida foi a de pedreiro, correspondendo a 16 casos (29,6\%), seguida da de marceneiro, com 9 casos (-Tabela 1), e 36 dos pacientes (66,7\%) tinham vínculo previdenciário no emprego, que garantia acesso a benefício previdenciário durante o período de afastamento, sendo que em 21 casos (38,9\%) ocorreu acidente de trabalho formal ( - Tabela 2). Quanto à escolaridade, 15 pacientes (27,8\%) tinham Ensino Fundamental completo ou incompleto, e somente 2 pacientes (3,7\%) tinham Ensino Superior completo (-Tabela 3).
Tabela 4 Número de dedos lesados

\begin{tabular}{|l|l|}
\hline Número de dedos lesados & N \\
\hline 1 dedo & 26 \\
\hline 2 dedos & 9 \\
\hline 3 dedos & 11 \\
\hline 4 dedos & 1 \\
\hline 5 dedos & 1 \\
\hline Proximais & 6 \\
\hline TOTAL & 54 \\
\hline
\end{tabular}

O lado esquerdo foi acometido em 83,3\% (45) dos casos. Considerando todos os tipos de lesão, 26 pacientes (48,1\%) tiveram lesão em somente 1 dos dedos, enquanto 6 pacientes $(11,1 \%)$ tiveram lesões proximais ( - Tabela 4$)$.

Levando em conta o total de lesões, o polegar foi mais envolvido, correspondendo a 30 casos (55,6\%), seguido do segundo dedo em 19 casos (35,2\%); o envolvimento do polegar também foi predominante nas lesões acometendo somente 1 dedo, em 18 casos (69,2\%), seguido do segundo dedo em 5 casos (19,2\%). Nas lesões envolvendo 2 dedos, a combinação com maior ocorrência foi a do polegar e do segundo dedo em 3 casos (33,3\%), seguida da combinação do terceiro e quarto dedos em 2 casos (22,2\%). Já nas lesões que acometeram 3 dedos, o envolvimento de polegar, do segundo e do terceiro dedos foi visto em 4 casos (36,4\%), assim como do segundo, terceiro e quarto dedos (-Figura 2 ) .

Foram identificadas amputações em 35 pacientes (64,8\%), sendo que 21 delas (60\%) envolviam o polegar, e $13(37,1 \%)$ não envolviam outros dedos. No total, 8 pacientes $(22,9 \%)$ tiveram de amputae o segundo dedo, mas somente em 2 deles (5,73\%) a lesão acometeu este dedo isoladamente. Todos os pacientes com amputação no terceiro, quarto e quinto dedos tiveram lesões em outros dedos.

Ao todo, 23 pacientes $(65,7 \%)$ foram submetidos a reimplante, sendo 3 macroreimplantes (13,7\%), 1 transmetacarpal e 2 no nível do punho. Outros dois pacientes foram submetidos a tentativa de reimplante, mas sem sucesso. Destes pacientes, 8 (30,4\%) necessitaram posterior regularização no decorrer da internação.

Com relação aos 25 pacientes submetidos a reimplante ou tentativa, o tempo decorrido entre a lesão e a entrada na sala cirúrgica variou de 4 horas e 40 minutos a 15 hotas e 15 minutos (média: 8 horas e 7 minutos; desvio padrão: 2 horas e 31 minutos), sendo $\geq 6$ horas para 21 pacientes $(84 \%$ ) ( - Figura 3 ).

Além das 35 amputações descritas, 3 pacientes (5,5\%) apresentaram lesões vasculares ( 2 foram submetidos a revascularização de dedos, e 1 tinha artérias irreparáveis), 10 $(18,5 \%)$ apresentaram lesões complexas com acometimento ósseo e de partes moles (fraturas expostas), e $6(11,1 \%)$ apresentaram ferimentos corto-contusos que acometeram somente partes moles (pele, tendões ou nervos).

Quanto ao horário do trauma, 14 lesões (25,9\%) ocorreram entre $14 \mathrm{~h}$ e $16 \mathrm{~h}, 12$ lesões (22,2\%), entre $12 \mathrm{~h}$ e $14 \mathrm{~h}$, e outros 10 casos (18,5\%), entre $10 \mathrm{~h}$ e $12 \mathrm{~h}$, totalizando 36 casos $(66,7 \%)$ entre $10 \mathrm{~h}$ e $16 \mathrm{~h}$ (-Figura 4). O mês de junho 


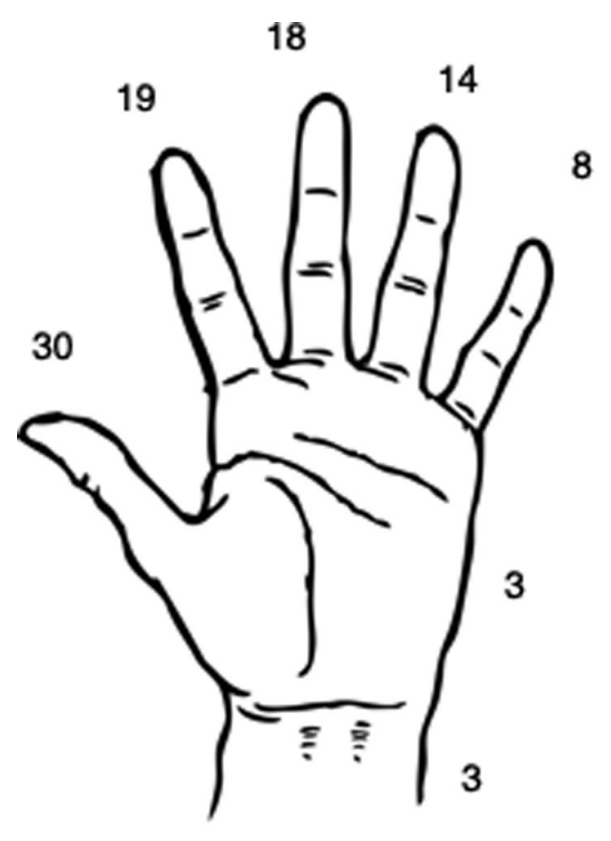

Total de lesões por dedo

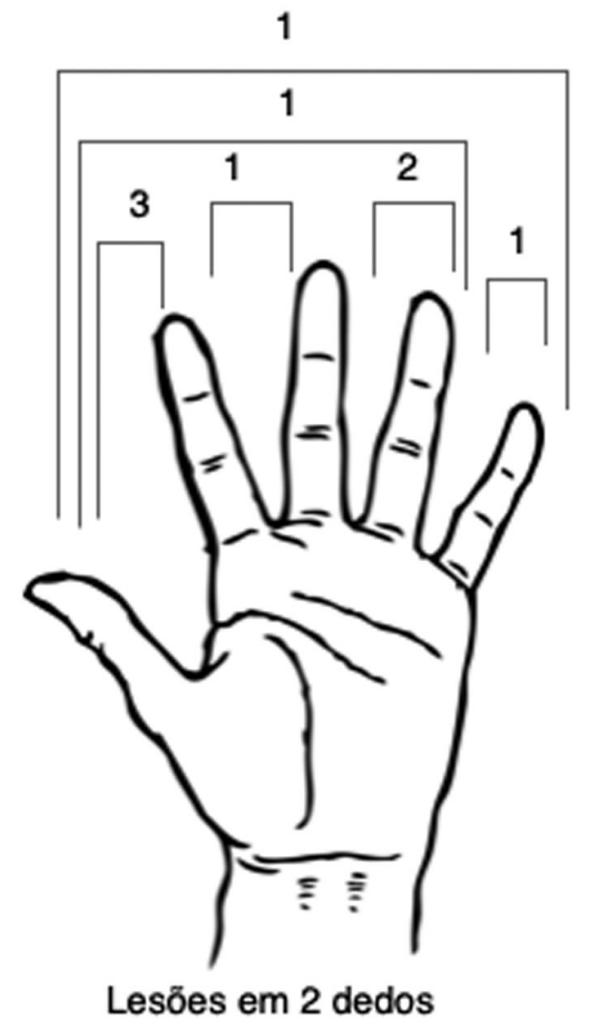

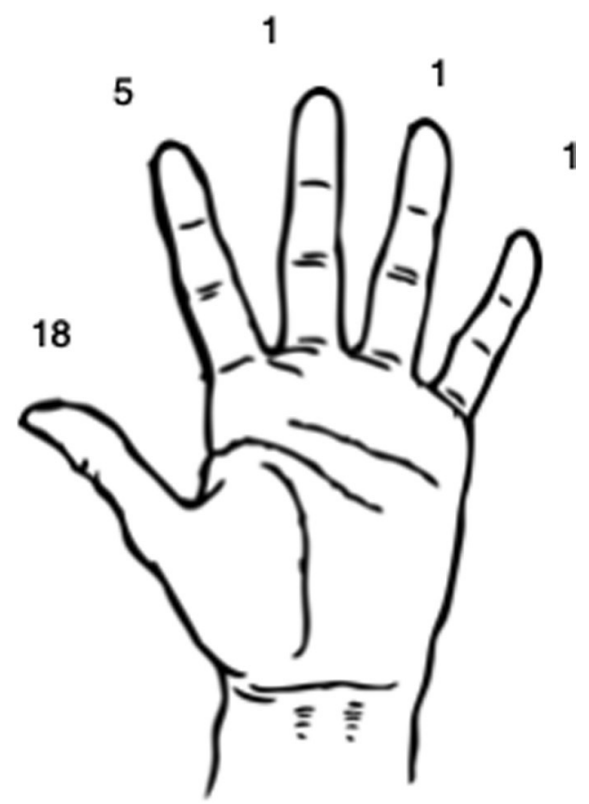

Lesões em dedo único

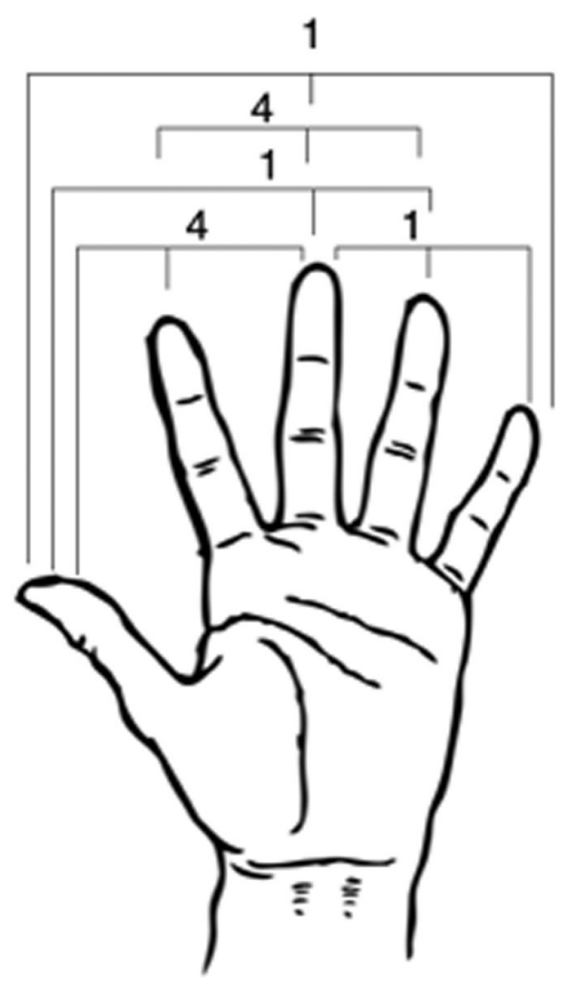

Lesões acometendo 3 dedos

Fig. 2 Número de lesões que acometeram dedo único, dois, ou três dedos.

apresentou o maior registro de ocorrências, com 13 casos $(24,1 \%)$ (-Figura 5).

\section{Discussão}

As lesões por serra circular ocorrem predominantemente no sexo masculino, possivelmente devido a associação com atividades de marcenaria, tipicamente exercidas por homens. Frank et al. ${ }^{2,3}$ descreveram uma amostra de 114 pacientes, sendo somente 8 do sexo feminino, ao passo que Selig et al. ${ }^{5}$ relataram uma predominância masculina com razão de $7: 1$, e Sabongi et al. ${ }^{6}$ relataram uma amostra que continha $98,1 \%$ de pacientes do sexo masculino. Fikry et al. ${ }^{1}$ e Hassine et al. ${ }^{4}$ relataram uma amostra exclusivamente 


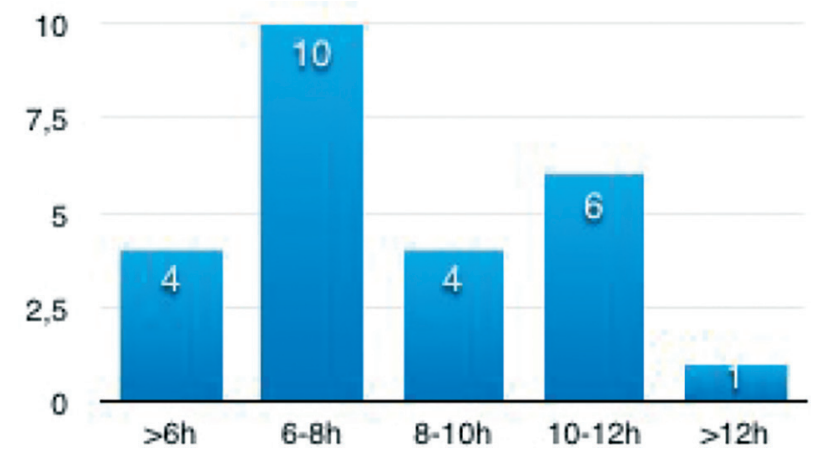

Fig. 3 Tempo decorrido entre o trauma e a entrada no centro cirúrgico.

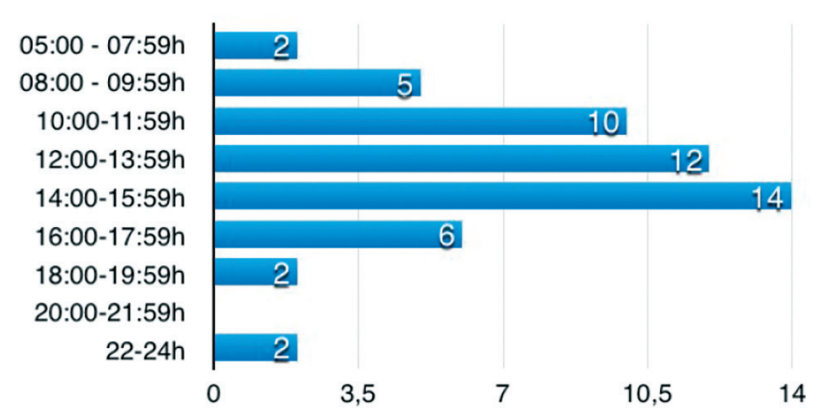

Fig. 4 Horário da lesão.

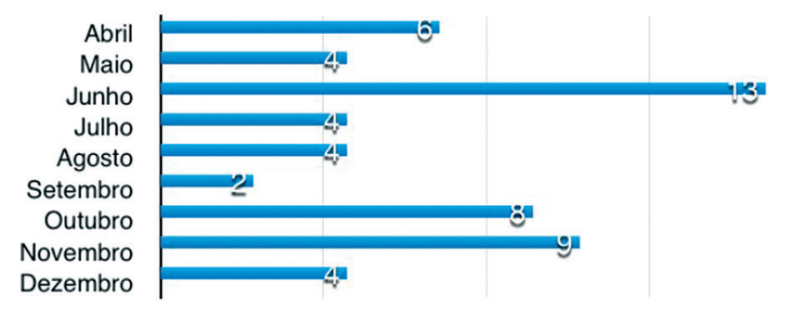

Fig. 5 Meses em que ocorreram as lesões.

masculina, semelhante ao que foi observado no presente estudo.

Frank et al. ${ }^{2}$ relataram idades entre 15 e 81 anos, com média de 49 anos, semelhante ao encontrado por Hassine et al., ${ }^{4}$ que descreveram idades entre 16 e 67 anos, com média de 31,24 anos. Estes autores, porém, descreveram a maioria das lesões em pacientes com menos de 30 anos. Selig et al. ${ }^{5}$ descreveram maior incidência em pacientes entre 45 e 64 anos e maiores de 65 anos, mas englobaram amputações por diversos mecanismos, embora a maioria das lesões tenha ocorrido por serra circular. No trabalho de Fikry et al., ${ }^{1}$ os pacientes tinham em média 25 anos (gama: 12 a 60 anos). Esta variação pode ser em decorrência do perfil socioeconômico dos usuários de serra circular em diferentes países. No presente trabalho, a maior parte das lesões (47 casos; 87\%) ocorreu em indivíduos entre 25 e 64 anos, concentrando-se principalmente entre 45 e 54 anos (19 pacientes; 35,2\%), semelhante ao encontrado por Frank et al. ${ }^{1}$ Supõe-se que este pico em idades mais avançadas possa ocorrer em decorrência da negligência em utilizar equipamentos de proteção e da sensação de confiança ao manusear o objeto; pacientes mais experientes relataram pular etapas de segurança devido à confiança excessiva. Já nos mais jovens, a falta de experiência pode ter um papel importante nos acidentes.

Devido ao baixo nível de escolaridade de grande parte destes pacientes, muitos acabam por ingressar em empregos informais, como evidenciado nos 38,9\% de acidentes de trabalho informais verificados neste estudo. Entre as atividades realizadas, são mais afetados trabalhadores da construção civil, o que indica que os riscos de acidentes ocupacionais nesses setores são consideravelmente altos em relação a outras atividades laborais, por exigir esforço físico e habilidade, o que expõe os trabalhadores a diversos riscos.

Fikry et al. ${ }^{1}$ relataram $88 \%$ de lesões acometendo o lado esquerdo, e Hassine et al., ${ }^{4}$ 64.61\%. Já Frank et al. ${ }^{2}$ encontraram 54 lesões na mão esquerda não dominante, e 49 na mão direita dominante. De modo similar, no presente estudo, encontramos $83,3 \%$ de lesões (45 casos) acometendo o lado esquerdo, um achado que corresponde aos descritos na literatura. A predileção desta lesão pela mão esquerda se justifica por ela estar mais próxima à lâmina da serra, segurando o objeto a ser cortado, e, portanto, é mais suscetível às lesões. Esse dado merece atenção para o planejamento de normas de segurança.

Hassine et al. ${ }^{4}$ identificaram amputações como as lesões mais comuns, em $85 \%$ dos casos, mas somente $5 \%$ envolvendo o polegar. Em nosso trabalho, diferentemente, as amputações foram menos frequentes ( $64,8 \%$ ou 35 pacientes), mas o polegar foi o dedo mais acometido (60\% ou 21 casos). A maior incidência de lesão neste dígito pode ser em decorrência da posição de pinça ao segurar o objeto a ser cortado.

Frank et al. ${ }^{3}$ descreveram amputações em 1 dedo em 16 casos, comparável aos 13 casos de amputação de polegar sem acometimento dos outros dedos encontrados em nossa casuística. Selig et al. ${ }^{5}$ descreveram reimplante em $28 \%$ dos casos de amputação, valor bem inferior aos $65,7 \%$ correspondendo a 23 pacientes encontrados em nossos casos. A única menção a macroreimplantes é feita por Frank et al., ${ }^{2}$ que descreveram um caso no nível da mão, semelhante ao encontrado por nós (um macroreimplante de mão e dois no nível do punho).

Lesões acometendo somente 1 dedo foram observadas em $48,1 \%$ dos casos no presente estudo (26 pacientes). Esse número condiz com o observado por Sabongi et $\mathrm{al}^{6}$, que relataram a maior parte das lesões em dedo único (50 casos), assim como Frank et $\mathrm{al}^{2}$, que neste estudo descreveu ainda, nas lesões de dedo único, 24 lesões no polegar e 14 no segundo dedo, o que condiz com nosso achado de predominância no lado radial, e com a combinação de polegar e segundo dedo sendo a mais encontrada em lesões acometendo 2 dedos. Além de ser o mais amputado, o polegar também é o dedo mais acometido em lesões isoladas.

Nas lesões em dois dedos, a combinação mais encontrada por Frank et al. ${ }^{2}$ foi a do polegar e do segundo dedo, semelhante ao nosso estudo, e nas lesões em três dedos, a combinação do segundo, terceiro, e quinto dedos. Outra combinação de três dedos relatada frequentemente foi a do terceiro, quarto e quinto dedos, que identificamos somente 
em um caso; no entanto, identificamos a mesma incidência de lesões no polegar, segundo e terceiro dedos, e no terceiro, quarto e quinto dedos. Já Hassine et al. ${ }^{4}$ descreveram lesões em múltiplos dedos em 77\% dos casos, com predominância de lesões no segundo e terceiro dedos.

Frank et al. ${ }^{3}$ descreveram uma maior incidência de lesões nos dedos radiais (polegar, segundo ou terceiro dedos), sendo um deles envolvidos em $88 \%$ das lesões em dedo único, e em $88 \%$ das lesões em 2 dedos. Esse achado é semelhante ao encontrado neste estudo, que identificou no total 30 lesões (55,6\%) envolvendo o polegar, 19 lesões $(35,2 \%)$ no segundo dedo e 18 (20,22\%) no terceiro dedo. Por serem os dedos radiais responsáveis pela pinça e preensão em oponência, sequelas nesses dedos podem levar a grande prejuízo funcional.

Quanto ao horário do trauma, a literatura descreve uma distribuição bimodal, com dois picos de incidência. Hassine et al. ${ }^{4}$ relataram picos de horário do meio-dia às $14 \mathrm{~h}$ (27 casos), e 27 casos entre 16 h e 18 h. Já Fikry et al. ${ }^{1}$ descreveram picos de incidência das $9 \mathrm{~h}$ às $12 \mathrm{~h}$ e das $13 \mathrm{~h}$ às $15 \mathrm{~h}$, e Selig et al., ${ }^{5}$ entre $10 \mathrm{~h}$ e $12 \mathrm{~h}$ e $14 \mathrm{~h}$ e $16 \mathrm{~h}$. Em nossa casuística, 26 lesões $(48,1 \%$ ) ocorreram entre $12 \mathrm{~h}$ e $16 \mathrm{~h}$, o que pode estar relacionado à sonolência pós-prandial. Outros 10 casos $(18,5 \%)$ ocorreram entre 10:00 e 12:00h, podendo ter correlação com cansaço ao final de um longo período de trabalho.

Em relação ao tempo decorrido até a entrada em sala cirúrgica, a média de 8 horas e 7 minutos pode comprometer o resultado do tratamento, pois, em sua maioria, trata-se de lesões com isquemia e necessidade de procedimentos vasculares, que idealmente necessitam pronta revascularização. Esse dado evidencia a deficiência do atendimento público, e é importante para a conscientização a respeito da necessidade da criação de centros especializados para o atendimento destas lesões, principalmente considerando que a maioria desses casos necessita de revascularização, cujo sucesso depende fundamentalmente da diminuição do tempo de isquemia do segmento amputado.

Selig et al. ${ }^{5}$ descreveram um pico de prevalência em maio, e um aumento da frequência entre agosto e novembro. Identificamos um pico de prevalência no mês de junho; no entanto, nosso estudo compreendeu somente nove meses. Não observamos fatores que possam justificar este dado.

A partir da descrição da população mais acometida por essas lesões, pode-se pensar em definir políticas públicas de prevenção na tentativa de reduzir seu número. Essas políticas só podem ser criadas entendendo os tipos de lesões mais frequentes, suas características, e os fatores que levam à sua ocorrência. Fikry et al. ${ }^{1}$ dividem os fatores de risco para as lesões por serra entre aqueles ligados à serra (falha do sistema de segurança, mais comumente removido pelo usuário, ou defeitos na manutenção, principalmente a falta de fio na lâmina), fatores da madeira (orientação das fibras e presença de nós), e fatores humanos (idade jovem, falta de qualificação e falta de atenção). Em nosso trabalho, não foi possível identificar o mecanismo que levou à lesão, mas o mecanismo de coice foi identificado na literatura ${ }^{2,6}$ como a principal causa, o que vai ao encontro dos relatos informais dos pacientes, que relatam um coice principalmente ao cortar através de nós da madeira. A hipótese de utilização incorreta da serra também deve ser considerada, pois é comum o uso de lâminas inadequadas à rotação de determinadas máquinas, bem como a retirada de mecanismos de proteção e a falta de uso de equipamentos de proteção individual (EPIs), também relatadas por Sabongi et al. ${ }^{6}$

No trabalho de Hassine et al., ${ }^{4}$ são descritos 170 pacientes em 8 anos (somente 130 incluídos no estudo) em um único serviço. Em um período de tempo semelhante, Frank et al. 2,3 identificaram em seu serviço 179 pacientes (114 pacientes incluídos no estudo). Obtivemos um número pequeno comparado aos desses trabalhos, mas, ao levarmos em conta o curto período de tempo, observamos uma frequência de lesões bem superior ao descrito na literatura. Por descrever um período de tempo recente, temos uma visão atual da realidade, que traduz o que está acontecendo neste momento em relação a estes acidentes.

Outras limitações deste estudo incluem o fato de ele ter sido realizado em um centro de referência em microcirurgia e reimplantes; portanto, o número de lesões mais graves será superestimado. Lesões menores, sem necessidade de abordagem por equipe especializada, acabam sendo tratadas em serviços de menor complexidade; portanto, estima-se que o número real destas lesões seja consideravelmente maior. Este estudo também se restringiu aos dados dos prontuários de atendimento, que não continham dados que poderiam ter relevância funcional (como dominância e nível anatômico da lesão). Tampouco foi realizada uma avaliação funcional dos pacientes após sua reabilitação.

\section{Conclusão}

As lesões por serra circular são muito frequentes em nosso meio e causam ferimentos graves, muitas vezes com sequelas permanentes. Características relacionadas ao período do dia em que as lesões ocorrem e à idade dos pacientes fornecem subsídios para o direcionamento de políticas de prevenção. Ficou bem caracterizado o padrão grave das lesões, com amputações, lesões complexas, e uma predominância nítida do polegar, o que revela a necessidade de no sistema público quanto ao atendimento emergencial para este tipo grave de lesão, com a criação de mais centros de referência capacitados para seu tratamento.

\section{Contribuições dos autores}

Cada autor contribuiu individual e significantemente para o desenvolvimento deste artigo. ALCF: redação do artigo e revisão de prontuários; LK: redação do artigo e busca de referências bibliográficas; KCA: coleta de dados e idealização da pesquisa; MRR: orientação, redação, e revisão do artigo.

\section{Suporte Financeiro}

Este trabalho teve suporte do Instituto de Pesquisa e Ensino Home, Brasília, DF, Brasil.

\section{Conflito de Interesses}

Os autores declaram não haver conflito de interesses. 


\section{Referências}

1 Fikry T, Saidi H, Latifi M, Essadki B, Zryouil B. La main traumatique par toupie: pour une meilleure prévention. Chir Main 2004;23 (02):96-99

2 Frank M, Lange J, Napp M, Hecht J, Ekkernkamp A, Hinz P. Accidental circular saw hand injuries: trauma mechanisms, injury patterns, and accident insurance. Forensic Sci Int 2010;198(1-3):74-78

3 Frank M, Hecht J, Napp M, et al. Mind your hand during the energy crunch: Functional Outcome of Circular Saw Hand Injuries. J Trauma Manag Outcomes 2010;4(01):11
4 Hassine YH, Hmid M, Baya W. La main de toupie: à propos de 130 cas. Tunis Med 2016;94(12):1-10

5 Selig HF, Nagele P, Voelckel WG, et al. The epidemiology of amputation injuries in the Austrian helicopter emergency medical service: a retrospective, nationwide cohort study. Eur J Trauma Emerg Surg 2012;38(06):651-657

6 Sabongi RG, Erazo JP, Moraes VY, et al. Circular saw misuse is related to upper limb injuries: a cross-sectional study. Clinics (São Paulo) 2019;74:e1076 